\title{
Impacto de un sistema ERP en la productividad de las PYME
}

\section{Impact of an ERP system on the productivity of SME}

\section{RONALd JAREd Romero REyes}

Ingeniero de Sistemas. Ingeniero de Soporte de la Universidad Sergio Arboleda.

Bogotá, Colombia. Contacto: ronald.rjr@gmail.com

\section{Sinndy Dayana Rico Lugo}

Ingeniera Industrial. Ingeniera de Producción de Mersen Colombia. Bogotá, Colombia.Contacto:dana.5179@gmail.com

\section{JuLIo Barón VeLÁndia}

Ingeniero de sistemas, especialista en Ingeniería de Software. Docente de la Universidad Distrital Francisco José de Caldas. Bogotá, Colombia.

Contacto:jbaron@udistrital.edu.co

Palabras clave: ERP, gestión, productividad, PYME, sistema.

Key words: ERP, management, productivity, SME, system.

\section{RESUMEN}

Teniendo en cuenta que la mayoría de las empresas en Colombia son del tipo PYME y que la toma de decisiones depende de la visión del fundador, lo cual puede dificultar la generación de avances en la apropiación de TIC (Tecnologías de Información y de Telecomunicaciones); los entes directivos y gerenciales de las empresas pueden solventar los problemas detectados en el sector
"Elaboración de Productos de Panadería y Bizcochería", apoyados en la utilización de diferentes soluciones tecnológicas, entre las que se encuentran los sistemas Enterprise Resource Planning (ERP), como formas de integrar y manejar la información de cada una de las áreas o departamentos de la empresa [1].

La disminución de los inconvenientes de las pequeñas y medianas empresas, y por ende el in- 


\section{investigación}

cremento de la productividad, puede ser abordado desde el desarrollo de un prototipo de software ERP que da respuesta a las siguientes preguntas: ¿qué características son relevantes en una aplicación informática para el apoyo en la gestión de recursos en las pyme del sector?, ¿de qué manera se debe gestionar la información para que esté disponible a las personas, según la responsabilidad y rol que asume en la estructura organizativa?, y ¿de qué forma una solución de este tipo influye positivamente en el incremento de la productividad de las PYME?.

\section{ABSTRACT}

Given that most companies in Colombia are SMEs and decision making depends on the founder's vision, (which makes it difficult to make progress when embracing ICTs) directors and management bodies of small enterprises may address the issues identified in the "Bakery industry" by strengthening their activities using different technological solutions. Enterprise Resource Planning (ERP) is one such solution, which works as a way to integrate and manage multiple-area information or information from various departments within the company [1].

Reducing the disadvantages of Small and Medium Enterprises (SMEs), and thus increasing their productivity is a problem that can be addressed by developing an ERP software prototype that attempts to answer the following questions: Which characteristics are relevant in a software application to support resource management in the SME sector?, what is the best approach to making information available to people according to the different responsibilities and roles within organizational structure?, and in what ways this type of solutions have a positive impact on SME productivity?.

\section{INTRODUCCIÓN}

Actualmente, el comportamiento de la economía en el Distrito Capital, en Colombia, tiene su base principal en las empresas de pequeño y mediano tamaño (PYME) y tiene en su territorio, aproximadamente, un $49 \%$ del total de empresas de este tipo existentes en el país [2]. Día a día, estas compañías sobreviven en el mercado presentando todavía problemas en relación a los modos y formas de gestión. Uno de los sectores representativos de esta problemática es el sector "Elaboración de Productos de Panadería y Bizcochería" que, al igual que otros sectores económicos, ha presentado una considerable fluctuación en lo concerniente a la creación y liquidación de sociedades, ventas, costos y competitividad.

Este artículo presenta la caracterización de una herramienta software desarrollada por los autores

y soportada en la integración de la Ingeniería Industrial y la Ingeniería de Sistemas, con el apoyo del Semillero y Grupo de Investigación Interoperabilidad Tecnológica y Semántica (INTECSE) de la Universidad Distrital Francisco José de Caldas, que permite la disminución de una de las grandes problemáticas de las PYME: la inadecuada toma de decisiones ocasionada por la falta de sistemas integrados de información que colaboren en la estructuración apropiada de la planeación, control y evaluación de los recursos empresariales, para que, finalmente, permita el incremento de la productividad.

\section{METODOLOGÍA}

En la figura 1 se observa la metodología seguida por los autores en el desarrollo del proyecto, cada número se refiere a un paso específico dentro del 


\section{investigación}

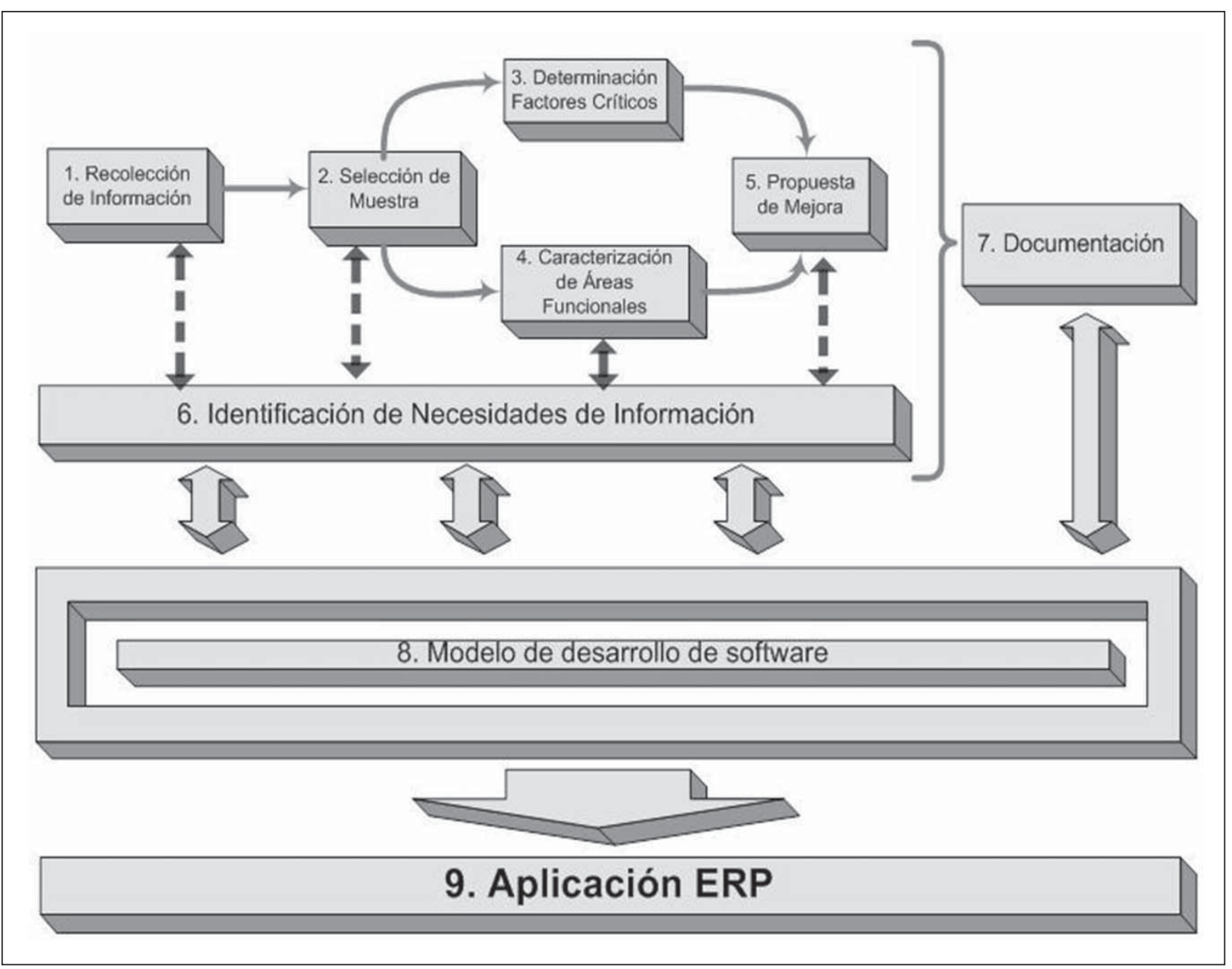

Figura 1. Metodología de trabajo.

Fuente: Tomado de [3]

proceso, pudiendo, algunos de ellos, ser ejecutados en forma iterativa y concurrente.

Paso 1: recopilación y revisión del material existente relacionado con el sector, con las PYME y con los ERP disponibles al momento del estudio.

Paso 2: basados en la revisión realizada, se evaluaron los diferentes tipos de muestreo existentes, con el fin de seleccionar adecuadamente las PYME muestra para el desarrollo del estudio, dadas las características de estas compañías y el tipo de tendencia de creación y disolución de sociedades en el sector.
Paso 3: determinación de los factores críticos del sector por medio del uso de matrices DOFA (Debilidades-Oportunidades-Fortalezas-Debilidades) a partir del modelo de las cinco fuerzas de Porter [4], realizando un análisis interno y del entorno de las compañías muestra, e identificando las decisiones que se toman frecuentemente en la administración. Esto generó la identificación de las problemáticas típicas del sector, sobre las cuales debería caracterizarse el ERP adecuado para alcanzar el incremento de la productividad.

Paso 4: de manera concurrente a la ejecución del tercer paso, se establecieron y caracterizaron las 


\section{investigación}

áreas básicas y funcionales de este tipo de empresas, diferenciándolas también por tamaño de empresa (micro, pequeña y mediana); por medio del uso adicional de diagramas de flujo de proceso, diagramas de recorrido, diagramas constructivos y demás herramientas propias de la ingeniería de métodos, tiempos y movimientos.

Paso 5: con los resultados de los pasos tercero y cuarto, se generó la propuesta de mejora de procesos, que incluyó el manejo de ITSGAS (acciones estratégicas generales en tecnológicas de la información) [4] que permitieron establecer la forma adecuada de atacar las problemáticas del sector por medio del uso de TIC's y, específicamente, de aplicativos ERP.

Paso 6: a lo largo de los pasos, del primero al quinto, se identificaron las necesidades de información que influyen en el proceso de toma de decisiones del sector por medio del uso adicional de ITSAS (acciones estratégicas en tecnologías de la información) [5], determinando así los requerimientos funcionales y no funcionales de un software ERP adaptado para el sector.

Paso 7: la metodología incluye el desarrollo progresivo y sistemático de la documentación, favoreciendo el acceso a la información en cualquier momento y manteniendo un registro tangible de los avances.

Paso 8: con el apoyo en los resultados de los pasos sexto y séptimo, y a lo largo de todo el proyecto, se realizó el prototipo de software ERP bajo el uso de un híbrido entre la metodología ágil de desarrollo XP (eXtreme Programming) [6], y los documentos entregables propuestos por la metodología de desarrollo RUP (Rational Unified Process) [7], de forma que los procesos y los resultados fuesen tangibles, mientras se mantenía un buen nivel de desarrollo de las funcionalidades definidas en el diseño.
Paso 9: finalmente, se integraron los módulos funcionales obtenidos y se realizaron las pruebas de sistema para verificar la cohesión y funcionalidad general que debe cumplir un aplicativo ERP, permitiendo incrementar la productividad de las PYME del sector de panadería y bizcochería, basado en la organización de la información, la reducción de recursos utilizados, el incremento de la producción y en las posibilidades que tienen estas empresas de adquirir un software de este tipo.

\section{RESULTADOS}

\subsection{Problemáticas del sector}

Como resultado del tercer paso de la metodología y basados en el libro Diseño de prototipo diagnóstico para la pequeña y mediana empresa, PYME. Enfoque mediante sistemas dinámicos [8], las problemáticas del sector se evidencian en temas como:

\subsubsection{Demanda variable}

La mayoría de las empresas no cuentan con clientes fieles; la demanda, es decir, los consumidores, aunque tienen preferencias, no se inhiben de comprar en otros sitios si se les facilita. Su patrón de compra depende de diferentes variables: calidad del producto, higiene, presentación, imagen corporativa, y la más determinante: el precio. Si las empresas no fortalecen los nexos que logran hacer clientes fieles, las debilidades y amenazas del mercado las dejan en una posición desventajosa.

\subsubsection{Desagremiación}

Un claro ejemplo de la resistencia de los empresarios del gremio a la asociatividad fue el estancamiento y casi fracaso de la Cámara Sectorial 
del Pan, creada por ACOPI, en el año 2008, grupo que buscaba detectar las problemáticas del sector e integrar soluciones, pero que, por la falta de afiliados, no llegó a buen término. A pesar de ello, se está comenzando el proceso de creación de la Asociación de Panaderos, ASODEPAN Seccional Bogotá, que pertenecerá a ASODEPAN Nacional. Con la constitución de esta asociación se busca agremiar a los empresarios de la panadería y la bizcochería de la capital con el fin de promover la adopción de nuevas estrategias (mercadeo, tecnologías, capacitación, precios, entre otros) que incrementen los niveles de utilidad, permanencia y crecimiento en el mercado bogotano.

\subsubsection{Estructuras heredadas}

Dado que el traspaso de conocimientos e información se realiza, casi exclusivamente, entre las generaciones dueñas de las PYME, no se presenta mayor incursión de nuevos conocimientos, principalmente si se habla de mejoras tecnológicas, esto debido al bajo nivel educativo de la gerencia y a la poca delegación de la toma de decisiones a personal de menor rango pero con mayores posibilidades de innovación y profesionalismo [9].

\subsubsection{Innovación y desarrollo tecnológico}

En las micro, pequeñas y medianas empresas se concentran los principales problemas de uso y apropiación de las TIC [10]. En estas circunstancias, se configura en el país una importante "brecha digital" entre las grandes, las pequeñas y medianas empresas, que se constituye, sin duda, en uno de los grandes retos para incrementar la productividad y la competitividad del país en los próximos años, aún más si se tiene en cuenta que las MiPYME representan el 98\% de las empresas de Colombia y que generan el $85 \%$ del empleo de la economía [11].
Esta problemática se encuentra representada por características como: las limitaciones del mercado de las PYME, que desestimulan la inversión en innovación y desarrollo; por los largos períodos de maduración y retorno incierto; la reticencia de la banca comercial a la financiación de la innovación y el desarrollo de empresas pequeñas; la insuficiencia de recursos blandos orientados hacia la innovación; la generalizada aversión de los empresarios hacia el riesgo; la informalidad en muchas de las PYME; la escasa tradición empresarial en materia de desarrollo y mejoramiento continuo de productos y procesos; y las pobres capacidades de gestión tecnológica en las empresas y otras instituciones.

\subsubsection{Comercialización y manejo de clientes}

Empezando por las deficiencias en la capacitación del personal que limita el correcto asesoramiento de los clientes (presentación del producto, asesoramiento al cliente y actividades de impulso y promoción), pasando por las debilidades locativas en distribución física de espacios de atención al público, la ubicación y aprovechamiento de los exhibidores, según su potencial de impulso; muchas PYME no adelantan acciones encaminadas a conocer cómo los están viendo sus clientes y qué les recomendarían para mejorar (estudios de mercado y mediciones de satisfacción, buzones de sugerencias).

\subsubsection{Sistema de gestión y aseguramiento de la calidad}

"E1 83,72\% adolece en absoluto de un sistema de gestión y aseguramiento de la calidad en la empresa" [9]. El concepto de calidad está erradamente identificado como la coincidencia del producto con los gustos y preferencias del empresario, administrador o representante en la empresa, y desconocen, casi en forma general, 


\section{investigación}

que es obligatorio ajustar el portafolio (productos y condiciones de servicio) a los gustos y necesidades del comprador.

Los sistemas de gestión y aseguramiento de la calidad observados en ciertas micro, pequeñas y algunas medianas empresas, no son en realidad un sistema integrado, por el contrario, es manejado en relación a controles al final de la línea de producción (normalmente en la operación de empaque), en ocasiones con márgenes de tolerancia relajados que dependen directamente del criterio del operario, lo que no influye positivamente en los niveles de competitividad de la compañía.

\subsection{Características de un ERP adecuado para el sector}

Teniendo en cuenta que la Planificación de Recursos Empresariales, o simplemente ERP (Enterprise Resource Planning), es un conjunto de sistemas de información gerencial que permite la integración de las operaciones de una empresa, especialmente las que tienen que ver con la producción, logística, inventario y costos; la integración de todos estos datos en una base de datos centralizada facilita la optimización de los procesos y la obtención de la información de manera más rápida y precisa, a la vez que permite a todos los usuarios compartir información y acceder a ella en forma constante. Además, debe contar con otras características que influyen en su diferenciación con otras aplicaciones, como: la división interna en módulos y la adaptabilidad, indispensables para el incremento progresivo de funcionalidades mediante el desarrollo y la incorporación de nuevos módulos.

Para el sector de estudio, primero, se deben considerar los factores críticos considerados de éxito para cualquier proyecto relacionado con tecnología que se muestran en la figura 2 :

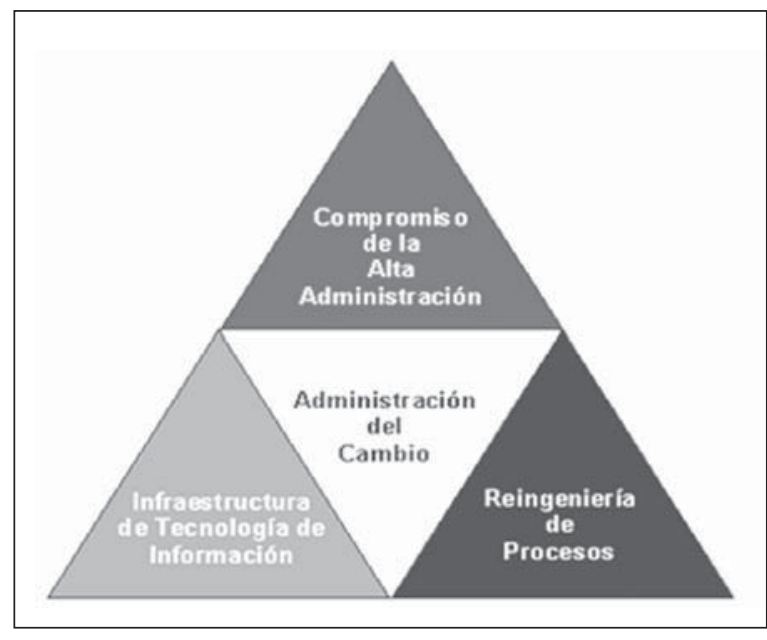

Figura 2. Factores críticos de éxito para proyectos ERP.

Fuente: tomado de [12]

En dicha figura se evidencia que las herramientas tecnológicas de hardware y software son apenas una parte de los aspectos a tomar en cuenta en un sistema ERP, teniendo mayor peso, en conjunto, aquellos factores relacionados con el aspecto humano y social. En torno a ello, para las PYME del sector de panadería, al igual que para la mayoría de las PYME colombianas, un sistema ERP debería constar en general de los módulos mostrados en la tabla 1.

Un buen sistema ERP para las pequeñas y medianas empresas del sector de panadería y bizcochería, debe ofrecer, adicionalmente, lo siguiente:

- Un grupo de aplicaciones firmemente integradas con los costos, que manejen la planeación agregada como base [13].

- Soporte local para consultoría e implementación, lo cual es crítico en tanto que las PYME, por lo general, no cuentan con suficientes recursos técnicos.

- Calcular los consumos de servicios públicos dependiendo del plan de producción planeado. 
Tabla 1. Módulos de un ERP.

\begin{tabular}{|l|l|}
\hline \multirow{4}{*}{ Módulo } & Submódulos \\
\hline \multirow{5}{*}{ Producción } & Gestión de productos \\
\cline { 2 - 3 } & Inventario \\
\cline { 2 - 3 } & Planeación de producción \\
\cline { 2 - 3 } & Pronósticos \\
\cline { 2 - 3 } & Administración proceso productivo \\
\hline \multirow{5}{*}{ Administración } & Costos de nómina \\
\cline { 2 - 3 } & Gestión de información del personal \\
\cline { 2 - 2 } & Gestión administrativa \\
\cline { 2 - 3 } & Administración cadena de abasto \\
\cline { 2 - 3 } & Administración de la demanda \\
\cline { 2 - 3 } & Configuración del software \\
\hline
\end{tabular}

Fuente: tomado de [3]

- Facilidades para modificar el horizonte de planeación de producción a un día.

- Generación de informes gráficos de fácil comprensión.

- Control de producción por operaciones que informe las bajas de producto.

- Opciones para mantener un seguimiento constante a la materia prima y sus rendimientos.

En la tabla 2, que se muestra a continuación, se visualizan algunos de los procesos críticos y sus porcentajes de mejora al hacer uso de un sistema del tipo ERP, hallados con el desarrollo del proyecto.

Como se observa en la tabla 2, para las empresas que formaron parte de la muestra seleccionada, el apoyo en la planeación y las herramientas de con- trol permitieron una reducción tanto en los recursos utilizados como en los costos asociados, aumentando en un alto porcentaje la productividad. Es importante resaltar que, para lograrlo, es necesario contar con el apoyo decidido y permanente de la parte directiva, tanto para mantener como para ampliar el uso de este tipo de aplicaciones.

\section{CONCLUSIONES}

El desarrollo de un sistema de gestión de operaciones basado en tecnologías ERP para las PYME de la ciudad de Bogotá D.C., en Colombia, permite a los propietarios y administradores de dichas empresas evaluar y mejorar sus procesos internos, si se cuenta con el compromiso de realizar el seguimiento adecuado de las características del software, así como su adecuada utilización.

El estudio y aplicación de mejoras en optimización de recursos, soportado en sistemas de información, incrementa el porcentaje de innovación y adaptación tecnológica de la PYME, permitiendo realizar una mejor planeación de los recursos empresariales.

El desconocimiento de la tecnología, por parte de los gerentes, es el principal obstáculo para la adopción de tecnologías de información y las comunicaciones en las pequeñas y medianas empresas.

Es necesario potencializar el desarrollo de sistemas ERP para las empresas del sector panadería y bizcochería e incrementar la formación tecnológica de sus propietarios.

\section{FINANCIAMIENTO}

Este artículo surgió a partir de la culminación del proyecto de grado denominado "Diseño y Desarrollo de un Prototipo ERP para las PYME del 


\section{investigación}

Tabla 2. Porcentajes de mejora.

\begin{tabular}{|c|c|c|}
\hline \multicolumn{2}{|l|}{ PROCESO CRÍTICO } & MEJORA \\
\hline \multicolumn{2}{|c|}{ Planeación de la producción y control de inventarios para tres días. } & \multirow[b]{3}{*}{$83.3 \%$} \\
\hline Sin ERP & Con ERP & \\
\hline $\begin{array}{l}12 \text { horas realizando las siguientes tareas: definir la } \\
\text { prioridad de los pedidos nuevos, revisar estado de } \\
\text { pedidos anteriores, revisar el inventario de materias } \\
\text { primas y de producto terminado, determinación de la } \\
\text { capacidad real de producción, asignación de materias } \\
\text { primas, revisión de tiempos de máquinas, asignación } \\
\text { de personal, envío de información al jefe de área }\end{array}$ & $\begin{array}{l}2 \text { horas utilizadas por el jefe de producción para ingre- } \\
\text { sar al sistema y para generar el plan de producción, } \\
\text { haciendo verificación y modificación manual de los } \\
\text { resultados. }\end{array}$ & \\
\hline \multicolumn{2}{|l|}{ Acceso a la información del recurso humano } & \multirow{3}{*}{$94 \%$} \\
\hline Sin ERP & Con ERP & \\
\hline $\begin{array}{l}0.28 \text { horas tarda una persona (ej. secretaría) en ir al } \\
\text { archivo a buscar la información del empleado y volver } \\
\text { al puesto de trabajo }\end{array}$ & $\begin{array}{l}0.016 \text { horas tarda una persona (ej. secretaría) en abrir } \\
\text { el sistema y en hacer la búsqueda del empleado }\end{array}$ & \\
\hline \multicolumn{2}{|l|}{ Definición de costos de producción de una semana } & \multirow{3}{*}{$96,5 \%$} \\
\hline Sin ERP & Con ERP & \\
\hline $\begin{array}{l}8 \text { horas dedicadas a las siguientes tareas: recopilar in- } \\
\text { formación, revisar consistencia y veracidad de los da- } \\
\text { tos, aplicar formulas de costos, realizar informe escrito }\end{array}$ & $\begin{array}{l}0.28 \text { horas utilizadas en ingresar al sistema y hacer la } \\
\text { búsqueda de los costos por fechas }\end{array}$ & \\
\hline \multicolumn{2}{|l|}{ Control de bajas (materias primas, producto terminado) } & \multirow[b]{3}{*}{$80,3 \%$} \\
\hline Sin ERP & Con ERP & \\
\hline $\begin{array}{l}0.66 \text { horas definidas de la siguiente forma: en un pro- } \\
\text { ceso completo de fabricación se realizan como míni- } \\
\text { mo } 8 \text { actividades, en cada una de ellas se revisan las } \\
\text { bajas y se registran en un formato en un tiempo pro- } \\
\text { medio de } 5 \text { minutos }\end{array}$ & $\begin{array}{l}0.13 \text { horas utilizadas para ingresar al sistema y digitar } \\
\text { las bajas }\end{array}$ & \\
\hline \multicolumn{2}{|l|}{ Generación de informes } & \multirow[b]{3}{*}{$95,8 \%$} \\
\hline Sin ERP & Con ERP & \\
\hline $\begin{array}{l}8 \text { horas invertidas en recopilar la información histórica, } \\
\text { en revisar consistencia y veracidad de los datos, en } \\
\text { ingresar los datos en un sistema para el manejo de } \\
\text { datos (ej. Excel) y en generar los documentos con los } \\
\text { reportes gráficos }\end{array}$ & $\begin{array}{l}0.33 \text { horas utilizadas para ingresar al sistema, llenar } \\
\text { los parámetros de generación de reportes y para ex- } \\
\text { portar los resultados a un archivo de texto }\end{array}$ & \\
\hline \multicolumn{2}{|l|}{ Actualización de la información de la empresa } & \multirow[b]{3}{*}{$83,4 \%$} \\
\hline Sin ERP & Con ERP & \\
\hline $\begin{array}{l}0.5 \text { horas utilizadas para realizar las actividades de } \\
\text { búsqueda de la información anterior, generación o } \\
\text { Ilenado de los nuevos formatos, archivado de los do- } \\
\text { cumentos }\end{array}$ & $\begin{array}{l}0.083 \text { horas utilizadas en ingresar al sistema, buscar } \\
\text { el registro, editar la información y guardar las modifi- } \\
\text { caciones }\end{array}$ & \\
\hline \multicolumn{2}{|l|}{ Toma de decisiones } & \multirow[b]{3}{*}{$95,8 \%$} \\
\hline Sin ERP & Con ERP & \\
\hline $\begin{array}{l}2 \text { horas es el tiempo promedio transcurrido mientras } \\
\text { una solicitud pasa por toda la cadena de mando y lle- } \\
\text { ga al gerente-dueño-empresa, y éste toma la decisión } \\
\text { frente a la situación }\end{array}$ & $\begin{array}{l}0.083 \text { horas son estipuladas para que la decisión sea } \\
\text { tomada, debido a que el sistema informa directamente } \\
\text { al gerente-dueño o al responsable directo de la ope- } \\
\text { ración a realizar }\end{array}$ & \\
\hline
\end{tabular}

Fuente: elaboración propia 
Sector de Panadería y Bizcochería" [3] avalado y aprobado por los proyectos curriculares de Ingeniería Industrial e Ingeniería de Sistemas de la Universidad Distrital Francisco José de Caldas, junto con la experiencia alcanzada a través del proyecto de extensión en ejecución "Acompaña- miento a las PYME del Sector en la Apropiación de las Tecnologías de la Información y las Comunicaciones (TIC) del Tipo ERP”, financiado por un valor de 6'900.000,oo por el Instituto de Extensión y Educación No Formal de la Universidad Distrital-IDEXUD.

\section{REFERENCIAS}

[1] Departamento Administrativo Nacional de Estadística DANE, Boletín de Prensa: Microestablecimientos Evolución 2007, [en linea]. Disponible: http://www.dane.gov. co/files/investigaciones/boletines/microestablec/Bolet_micro_IIItrim07.pdf

[2] A. Shtub and R. Karni, ERP: The Dinamics of Supply Chain and Process Management. Israel: Springer, 2010.

[3] R. Romero y S. Rico, Diseño y Desarrollo de un Prototipo ERP para las PYME del Sector de Panadería y Bizcochería, [Tesis Pregado], Bogotá: Universidad Distrital Francisco José de Caldas, 2010.

[4] J. Amaya, Gerencia, Planeación y Estrategia: Fundamentos, Modelo y Software de Gestión, Bucaramanga: Universidad Santo Tomás, 2005.

[5] R. Andreu y J. Valor, Estrategias y Sistemas de Información, Barcelona: Mc Graw Hill, 1996.

[6] K. Beck, Una Explicación de la Programación Extrema, España: Addison-Wesley Iberoamericana España, 2002.

[7] I. Jacobson, G. Booch y J. Rumbaugh, El Proceso Unificado de Desarrollo de Software, Addison Wesley Publishing Company, 2004.
[8] G. Méndez y L. Álvarez, Diseño de prototipo diagnóstico para la pequeña y mediana empresa, PYME. Enfoque mediante sistemas dinámicos, Bogotá: Fondo de $\mathrm{Pu}-$ blicaciones Universidad Distrital Francisco José de Caldas, 2004.

[9] SENA, [Regional Valle], Caracterización Ocupacional Industria de la Panificación y la Repostería (Actualización), Buga: Servicio Nacional de Aprendizaje, 2006.

[10] Cámara de Comercio de Bogotá, Adopción y Uso de TIC 2009, [en línea]. Disponible:

http://www.ccb.org.co/publicaciones/769/ Adopción yusodeTIC_ult.pdf

[11] M. Rodríguez, "El Plan Nacional de TIC2008 - 2019”, Asociación Colombiana de Ingenieros de Sistemas. 2009, [en línea]. Disponible: http://www.acis.org.co/filead$\mathrm{min} /$ Revista_104/columnista-invitado.pdf. Septiembre 2009

[12] J. Guerrero, "ERP al Alcance de las PYMES”, Gestiopolis, junio, 2009, [en línea]. Disponible: http://www.gestiopolis. com/recursos/documentos/fulldocs/ger1/ erppymes.htm

[13] J. Torres, Planeación Agregada en la PYME, Bogotá: Editorial UD, Universidad Distrital Francisco José de Caldas, 2006. 